\title{
Emotional Healing in Cancer Patients
}

\author{
Michael D. Winer \\ Human Potential Unlimited, Ltd., Portland, OR, USA
}

\section{Rationale for Developing an Emotional Healing Process}

Emotions play a central role in our lives, providing the basis for many of our responses to events as well as enriching the depth and breadth of our experience. Whether the emotion is desire, excitement, fear, curiosity, aloneness or creativity, emotions stimulate our reactions and provide the motivation for many of our activities.

Emotions affect more than just our mental and emotional states; they affect our physical bodies as well. Our bodies respond to joy and sadness, fear and courage, worry and certainty, security and insecurity. When we are healthy, our emotions flow through us without obstruction. However, when there is dysfunction, there is a blockage of their flow. Whether the blockage involves a limitation in the flow of a positive emotion or a fixation on a negative emotion, any blockage creates impaired function. As a result, emotional blockage and fixation is one basis for physical problems and health issues. Emotions contribute beneficially in creative and expansive ways when in their healthy state, while causing disease and impairment in their blocked or dysfunctional state.

Since there is a normal and natural movement through every aspect of our experience when we are in a healthy state, in its broadest sense, healing is about movement. It is about having our experience without attachment. In order to maintain and evolve our health, we want to experience movement through every aspect of our experience. Therefore, when disease or dysfunction is present, we want to initiate movement through our experience to resolve every fixation and blockage. This is equally true whether the disease or dysfunction exists on a physical, emotional, or mental level of our experience.

There are many different aspects of our experience, and we require a way to address each of those aspects. We need a means to evolve our thoughts, emotions, personalities, physical bodies, physical movement and coordination, communica- tion, intention, intuition, instincts, and other aspects of our experience. In this context, we require an emotional healing process because that is the only way to evolve our emotions. We cannot think our way around them, and we cannot ignore them or wish them away. We must feel our emotions and move through them in order to heal them.

In addition, an emotional healing process is a very effective method for evolving every other aspect of our experience. The skills that we master in learning to heal our emotions are practical for evolving and resolving any issue. Thus, while the elements of our experience seem to be diverse and unrelated, the process of emotional healing is equally useful in dealing with mental, physical, and emotional issues.

\section{The Emotional Healing Process}

There are five steps involved in an effective emotional healing process. The steps are as follows:

1. Become aware that an issue exists. 2. Focus on the emotion of the issue. 3. Separate from the trigger and refocus on the emotion. 4. Relax through the emotion. 5. Let go of the emotion or feeling.

While we do not have to take all of these steps consciously, they are the steps that we must go through to achieve healing. While some may seem obvious, it is important to acknowledge them so that we have clarity about what we are doing and so that we can teach others for whom they are not obvious.

First of all, in the context of this work, no healing can be accomplished without awareness. Whether the physician is aware of the patient's issues to guide him through his issues or the patient is aware of his own issues, someone must have awareness for this process to be effective. We must be aware that an issue exists before we can focus and direct our healing efforts. Thus, the first step involves paying special attention to

\begin{tabular}{ll}
\hline KARGER & @ 2007 S. Karger GmbH, Freiburg \\
Fax +49 7614520714 & Accessible online at: \\
$\begin{array}{l}\text { E-mail Information@Karger.de } \\
\text { www.karger.com }\end{array}$ & www.karger.com/brc
\end{tabular}


our experience to notice when an issue is present. This skill will continue to develop and improve as we practice our emotional process and become more attuned to how we relate to the world. Once we have awareness of an issue, we want to focus on the emotion associated with it. This requires that we step back from our experience in the moment and simply focus on the emotion that we are feeling. Without focus, we will not be able to achieve the emotional movement that we require to accomplish healing.

Next, we consciously separate from the trigger of our emotion and move our focus to the emotion itself. The ability to separate is vital to the emotional process. It is common for people to direct their emotions and issues at whomever or whatever triggered them. However, focusing on the trigger does not resolve our emotional issues. Instead, we want to shift our focus off of the trigger and onto our emotional response. We need to remain focused on the emotion itself because that is where the healing has to occur. We may not be able to control the trigger, but we can certainly heal our emotional response. By healing our emotions, the trigger will no longer have the same effect on us.

The movement through an emotion and the resultant healing is accomplished using relaxation. Once you are focused on an emotion or feeling, the next step is to simply relax into it and through it to achieve healing. Be careful not to go too deeply or too lightly into the emotion. Just feel the emotion and try to stay on a middle ground with it. This step may seem difficult at first but will become more easily navigated with practice. In addition, I recommend that people start with milder emotions first so that they are better prepared when more difficult emotional issues present themselves.

Lastly, we must let go of the emotion or feeling. The step of letting go is actually a continuation of the relaxation step in that we persist in relaxing through the feeling until it completely releases and we have let go of the issue. By following these steps, everyone can eventually resolve his or her issues and enhance the energetic movement associated with health and well-being. Keep in mind that healing is a continual process and that your emotional process will be something you utilize throughout your life to achieve and maintain a healthy state of being.

\section{Applying Our Emotional Healing Process}

Emotional healing has a critical role to play in the health care arena in conjunction with whatever other approach one chooses to use to address a particular health concern. We can apply the emotional healing process in several ways to have an immediate impact on our patients' health. We can use the process to assist our patients with the issues that cause their health concerns. We can assist patients in dealing with their response to their conditions as well as how they handle the responses from those with whom they interact. We can teach the process to our patients so that they can take better care of themselves. And we can use the process ourselves to improve our health and to make us better physicians.

\section{Assisting Patients with the Emotional Causes of Their Health Concerns}

If we look beneath the physical, physiological, and pathological issues of our bodies, we can explore the relationship between emotion and disease. While it would be convenient to have a formula for the relationship between specific emotional issues and diseases, there is a lot of variability in our patients and in the issues that contribute to their conditions. Thus, while there are some generalities to look for, everyone must be addressed individually. Everyone has their own individual makeup and responds to their experience uniquely.

Many of the patterns involved with cancer, as well as with other diseases, are not readily visible or necessarily conscious to the person who is experiencing them. We may have to take a very thorough family and personal history to determine any emotional foundation for the disease that is present. Things to look for include a history of abuse, not feeling loved, grief, loss, not feeling cared for or cared about, invalidation, rejection and worthlessness. In addition, we want to look for feelings of nonacceptance, self-hate, guilt, and self-judgment. We also need to ascertain what, if any, emotional trauma occurred. While many traumatic events are defended to the point of being unconscious, with experience, it is possible to perceive trauma that exists in another person's body and being. It may also be necessary to probe people's histories to assist them in remembering issues that are present.

Looking at our observations and research from the perspective that emotional issues contribute to disease and dysfunction offers valuable insight and understanding. For instance, from this perspective, a plausible explanation for the recurrence of cancer is suggested as resulting from incomplete resolution of the underlying emotional issues. Thus, the observation that surgery, radiation and chemotherapy can facilitate the recurrence of breast cancer and its spread to the lungs, mediated in part by the compound TGF-beta, may be explained by the continuing presence of the causal issues [1]. The body is simply expressing the emotional issues that have not been adequately addressed.

An equally important aspect of our healing work involves the evolution of the positive qualities that nurture health and well-being. Typically, people do not do any better at allowing positive emotions to flow through them than they do with letting go of negative emotions. Thus, a beneficial exercise for all of our patients is to have them focus on the positive qualities that they desire and to relax through those feelings as well. Do they want love, acceptance, validation or nurturing? By having our patients focus on the desired feelings, the feelings flow through their bodies to support their healing.

The ultimate goal of working with positive qualities is to be able to give one's self the feelings of love, acceptance, and va- 
lidation and not to look outside oneself for these feelings. We open the door to disease when we do not master that the source of love, acceptance and validation is within us. By cultivating the flow of these feelings through our bodies and our being, we bring incongruent emotions to awareness and make our bodies and our beings into less hospitable environments for disease and dysfunction. It is not adequate to simply perform this as a mental exercise. We must truly feel the love and acceptance flowing through us.

\section{Other People's Issues as a Source of Disease}

Our bodies can readily absorb the issues of other people when we open to them. We open to other people when we look for feelings or emotions from them instead of giving it to ourselves and when we have blocked emotions that we need to heal. Once other people's issues are in our space, our bodies respond to the emotion as if it is our own. And if the issues are significant enough, they can cause disease and dysfunction. A great deal of disease and dysfunction results from ineffective separation from other people's issues.

One common avenue through which people direct their issues at others is through abuse. Whether the abuse involves direct physical or sexual abuse, or is the result of being the object of demeaning behavior, it can inflict deep pain and wounds that require healing to reclaim normal physical, physiological, psychological, emotional, and social function. Abuse can have a deep and lasting effect on the people who are the targets of the abuse, and cancer is one way that abuse can manifest itself in our bodies.

One of the most important skills that we can learn and master is how to effectively separate from other people's emotions. Once we understand the significance of this problem and the impact it has on our health, we can work on the challenge of recognizing a particular issue as someone else's. In general, this is not difficult. When we attempt to heal an emotion by relaxing through it, we will be able to attain healthy movement and release when the issue is our own. However, when the issue is someone else's and we attempt to move through it, we will become stuck in it and will not achieve the desired movement. Thus, when we try to relax through a feeling or emotion and do not experience movement, it is most likely due to the issue being someone else's. In the event that we are dealing with someone else's issue, our task is to separate from their issue. To do that, we must become aware of the way that we engaged with it. This can be quite challenging because it is often a pattern of interacting with other people with which we identify and, therefore, are not aware that it is a problem. Typically, it involves opening to others for something that we want, such as care, love, acceptance, approval, worth or validation, which then pulls others' issues into our space. Negative emotions, such as feeling unworthy, insecure, responsible for others, uncared for or invalid, can open us to other people as well. These are the issues on which we will want to focus to separate from other people's issues.

\section{Assisting Patients with Their Issues about Their Health Concern}

An emotional process is essential for assisting people in dealing with their issues in relation to their disease or disorder. Anxiety, depression, fear, hopelessness, pain, uncertainty, and powerlessness can be easily recognized and defused so that the patient has the best chance for successful treatment and outcome. Ideally, we can guide our patients through these feelings to resolve them more permanently. And we want to help our patients develop their courage, strength, hope, and ability to respond to their own needs to facilitate the healing process. We can support our patients by nurturing these positive qualities in addition to using our emotional process to resolve any issue that arises in reaction to their fears, illness or disease. We need to engage people's best attitudes and cooperation to get the most out of the care that we provide.

\section{Assisting Patients with Other People's Responses to Their Condition}

Another important use for our healing process is in helping our patients deal with the issues and responses of their friends and families. How those people respond to our patients' issues can be critical to the support they receive and the outcome of the therapy. In this context, when someone has a health issue, it can affect their relationships and open them to the issues of the other people in their lives. Other people's issues can be obstacles to progress and can even become an overwhelming burden that adds to the original health concern.

We can assist our patients and their families and friends so that the influence of significant others is beneficial rather than contributing to the burden and challenge faced by our patients. This will typically necessitate guidance for how our patients interact with other people and, potentially, encouraging those significant others to become more involved in our patient's healing so that they bring support for the healing rather than projecting their own issues of pain, loss, grief or fears onto the situation. People coping with diseases have enough to handle without having to wrestle with other people's issues as well.

Of course, many health issues develop in direct response to the people around us. The issues that develop in relationships and the stress that they can create often contribute to the physical, mental, and emotional state of everyone involved. When the other people in a person's life contribute to his or her disorder, those relationships will need to be addressed to achieve the healing that we desire.

\section{Using a Healing Process to Improve Our Personal and Professional Lives}

We, the physicians, can heal ourselves to improve our own health and well-being and to complement our professional skills. By pursuing our own healing, we can reduce our stress, bring fewer issues into the workplace, and improve our enthusiasm and mental state. Developing our emotional healing process 
will also give us a better understanding of our clients and contribute to the development of the skills for how to approach people's health issues. The more practice we have with our own healing, the easier it is to recognize and understand other people's issues and to guide them through those issues, if we choose.

Patients come to us with a variety of problems and in a variety of different states of consciousness and responsiveness. A person's emotional state can tell us a lot about what to expect in their response to care. Some want us to take care of their issues, while some want to be more involved in their process. Some are open to what we are saying, and some will be resistant. Some will be feeling hopeful, and others will be feeling hopeless. Some will feel courageous, and others will be consumed by fear. Some will believe that they can heal, while others will feel that healing is impossible. The better our understanding of emotions and emotional healing, the more accurately we can assess our patients' conditions and ability to deal with medical procedures. This will foster better patient communication, trust, understanding, cooperation, and compliance. We will also be able to respond more appropriately to our patients, which will enhance the success of our therapeutic intervention.

Our patients open to us to get information. They are looking for any insight or nuance that tells them what we really think and feel about their condition and their prognosis. They open to understand our perspective and observations. As a result, we have tremendous power to influence the outcome of our care through the manner and emotion that we bring to the doctor-patient interaction. Patients are often fearful, and this is supported when health care personnel have their own fears. Fear, uncertainty, powerlessness, hopelessness, and uncaring are communicated to the patient just as well as confidence, hope, care, certainty, and respect. There is no benefit to expressing our worst fears without the proper balance of presenting a more complete picture. We can engage our patients' courage, determination, desire to actively participate in the process, and ability to heal in part due to our own emotional state, through how we interact with them and through how we impart our knowledge and information. As physicians who want to mobilize the best response in our patients, we can encourage them to become partners in whatever method is applied to the evaluation, diagnosis, treatment, and prognosis.

If we have issues around a particular condition, we can easily communicate our feelings to our patients consciously or unconsciously. It is easy to fall into thinking of the expected disease outcome and progression when patients are not responding to medical intervention. To be more successful in our health care practices, we must come into our power about people's ability to heal even the most insidious and difficult conditions. Rather than be defeated by the prognosis, we can inspire our patients to rise to the occasion and do the unexpected. Sometimes even the most difficult cases can be resolved.

\section{Conclusion}

Our bodies respond to emotion, and the seeds of disease and dysfunction are sown by unresolved issues. By expanding the scope of what we address through our health care, we can address the emotional contributions to disease. Using an effective emotional healing process in conjunction with other medical intervention is essential to achieve the best results for our efforts on our patients' behalf.

\section{Reference}

Biswas S, Guix M, Rinehart C, et al.: Inhibition of TGF- $\beta$ with neutralizing antibodies prevents radiation-induced acceleration of metastatic cancer progression. J Clin Invest 2007;117:1305-1313. 